\title{
Spinal Cord Compression with Occult Bony Fragment in Osteoporotic Vertebral Fracture: A Case Report
}

\author{
Yoshinori Ishikawa, Naohisa Miyakoshi, Michio Hongo, Yuji Kasukawa, Daisuke Kudo and Yoichi Shimada \\ Department of Orthopedic Surgery, Akita University, Graduate School of Medicine, Hondo, Japan
}

\begin{abstract}
Keywords:
vertebral fracture, occult fragment, spinal cord compression, late collapse, anterior spinal artery syndrome, dynamic CT

Spine Surg Relat Res 2021; 5(4): 310-312

dx.doi.org/10.22603/ssrr.2020-0106
\end{abstract}

Osteoporotic vertebral fracture is a common injury that can be treated conservatively; however, vertebral collapse with retropulsed bony fragment caused by unstable nonunion rarely persists ${ }^{1}$. Approximately $12 \%-13.5 \%$ of the cases exhibit failure of conservative treatment and show neurological deterioration requiring surgical treatment ${ }^{2,3)}$. We present a rare case of undetected spinal cord compression by an occult mobile bony fragment and discuss how to avoid overlooking and neurological deterioration.

An 84-year-old woman with Sjogren's syndrome injured her T12 vertebral fracture after a fall and was conservatively treated using thoracolumbosacral orthosis (Fig. 1). However, diffuse bilateral lower extremity weakness (absent reflexes without sensory or urinary disturbances) progressed for two months. Magnetic resonance imaging (MRI) revealed an old vertebral fracture with an effusion at T12, with no spinal cord compression (Fig. 1). Anterior spinal cord syndrome was diagnosed after referring to a neurologist; thus, conservative treatment with rehabilitation was started. Nevertheless, she was referred to us four months after her fall because of back pain, an inability to stand, and motor weakness (hip flexion, knee extension, ankle dorsiflexion, and plantar flexion: $3+/ 3,4 / 4-, 1-/ 1-$, and $2 / 2$, respectively). Bilateral paresthesia below the L4 level was recorded along with residual urine. Neuromuscular disease was excluded based on an axonal pattern of nerve conduction study and negative albuminocytologic dissociation of the cerebrospinal fluid. A computed tomography (CT) myelogram showed a wide canal in the supine position; however, a dynamic CT in the flexion position revealed spinal cord compression with a mobile bony fragment (Fig. 2). She underwent laminectomy, vertebroplasty with hydroxyapatite block, and posterior spinal fixation from T10 to L2 (Fig. 3). Pain relief enabled her to stand with support postoperatively. Her motor weakness also improved but not completely (hip flexion, knee extension, ankle dorsiflexion, and plantar flexion: 4/4, $5 / 5,2 / 1$, and $3 / 3$, respectively). She could walk with canes at eight months. CT revealed a complete fusion of the bony fragment within the vertebra one year later (Fig. 3). Although she had motor weakness with ankle dorsiflexion of $3-/ 2$, she could walk without aids and perform activities of daily living (ADL) independently at two years.

The incidence of fall-related vertebral fracture is not well known because osteoporotic fracture occasionally occurs asymptomatically. Fall-related vertebral fracture is seen in $33 \%-50 \%$ cases that show vertebral fractures radiologically ${ }^{4,5}$. Conservative treatment with spinal orthosis is effective in $86.5 \%-88 \%$ of patients ${ }^{2,3}$. However, patients with neurological deterioration and/or severe pain with delayed union require surgery. Although X-ray is the first line of examination and flexion/extension examination is relatively simple, achieving an accurate diagnosis is difficult. Thus, failed conservative treatments require MRI evaluation, which is normally performed in the supine position and reduces the collapsed vertebra. Nonobvious compression of the spinal cord leads to neuromuscular disease or ischemic changes in the spinal cord. Anterior spinal artery syndrome characterized by paraplegia, sensory dissociation, and bladder disturbance is induced by ischemic changes related to arterial flow, with the extent of the disorder varying case by case. Myelitis and polyneuropathy are also rare but had to be excluded in our case, as $20 \%$ of Sjogren's syndrome cases can be associated with central nerve system involvement ${ }^{6)}$. The absence of spinal cord compression led to the misdiagnosis.

Corresponding author: Yoshinori Ishikawa, isikaway@doc.med.akita-u.ac.jp

Received: May 30, 2020, Accepted: July 7, 2020, Advance Publication: August 31, 2020

Copyright (C) 2021 The Japanese Society for Spine Surgery and Related Research 

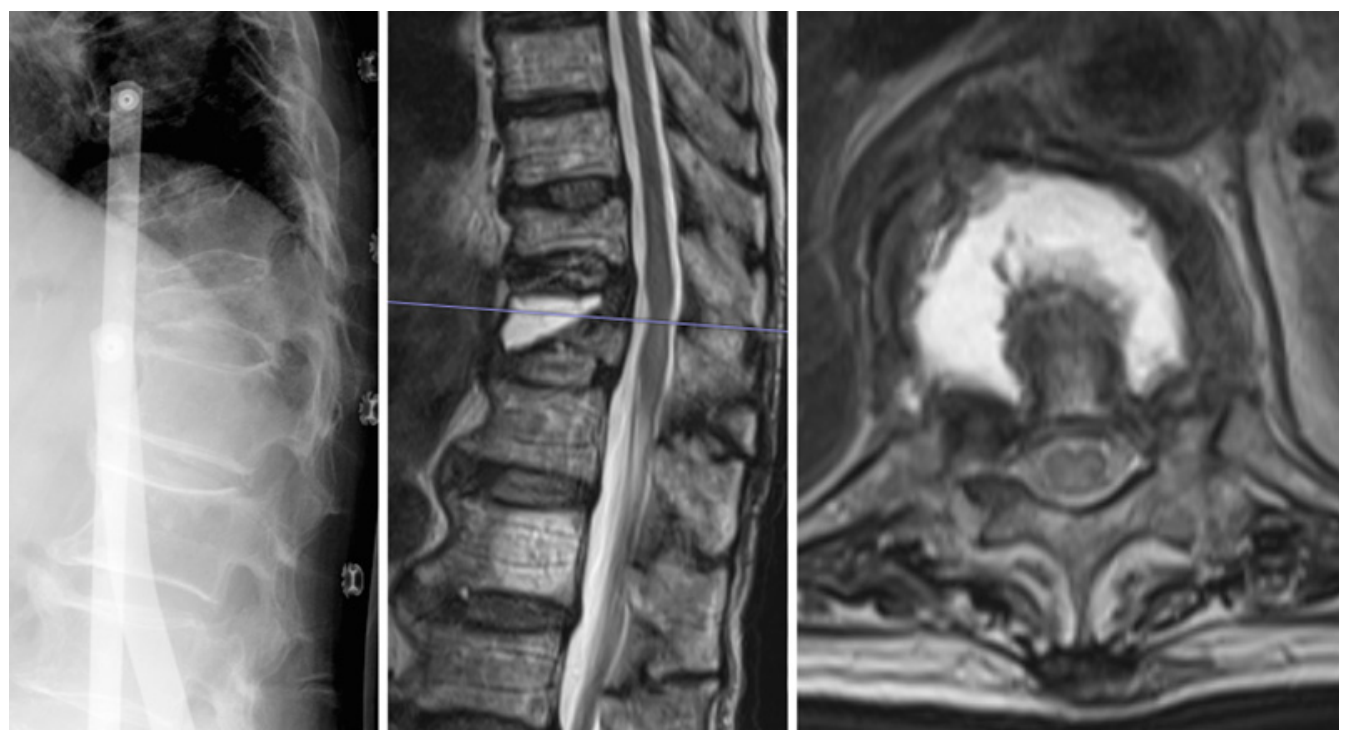

Figure 1. X-ray (left) showing a vertebral wedge fracture; MRI (middle, right) showing an old vertebral fracture with effusion without spinal cord compression.
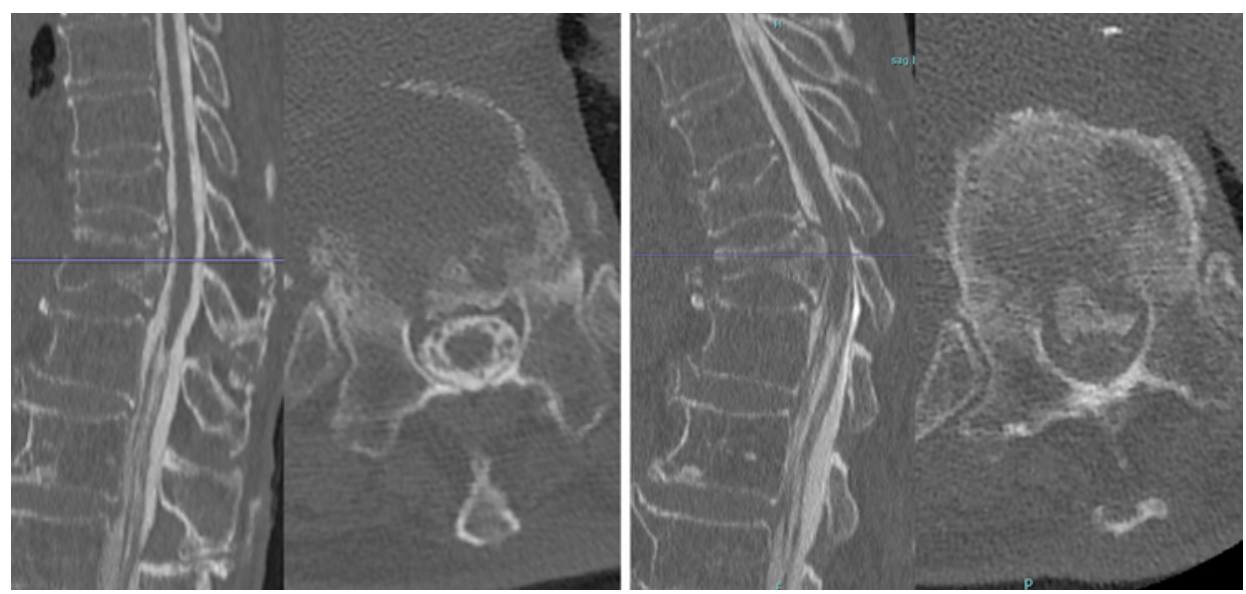

Figure 2. CT (left) in the supine position showing no compression of the spinal cord; however, flexion dynamic CT (right) reveals protrusion of occult bony fragment and spinal cord compression.
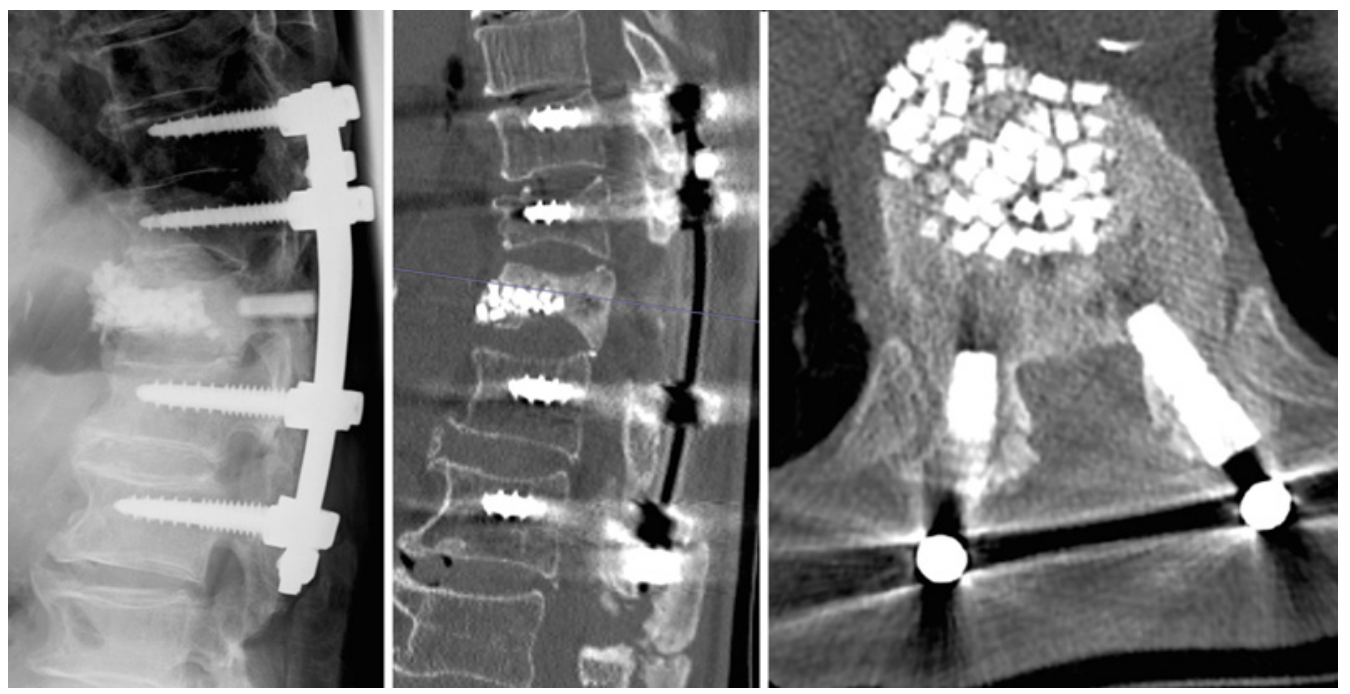

Figure 3. Postoperative X-ray and CT revealed rigid fixation and complete fusion of the bony fragment within the vertebra one year later. 
Risk factors of an occult bony fragment might be large cavity, filled fluid, and partial fracture of posterior vertebral wall. Fluid without gas suggesting a complete closed space, and fluid pressure efficiently affects the movement of bony fragment. Clinicians should be aware of the possibility of bony protrusions with dynamic spinal motion. An understanding of the pathogenesis enables an early diagnosis and the prevention of deterioration, which along with appropriate treatment prevents the deterioration of ADL.

Conflicts of Interest: The authors declare that there are no relevant conflicts of interest.

\section{Sources of Funding: None.}

Acknowledgement: We thank Enago (www.enago.jp) for editing a draft of this manuscript.

Author Contributions: Y. Ishikawa wrote and prepared the manuscript, and all of the authors participated in the study design. All authors have read, reviewed, and approved the article.

Ethical Approval: Unnecessary.
Informed Consent: Written informed consent from the patient was obtained prior to submission.

\section{References}

1. Kempinsky W, Morgan P, Boniface W. Osteoporotic kyphosis with paraplegia. Neurology. 1958;8(3):181-6.

2. Shikata J, Yamamuro T, Iida H, et al. Surgical treatment for paraplegia resulting from vertebral fractures in senile osteoporosis. Spine. 1990;15(6):485-9.

3. Tsujio $\mathrm{T}$, Nakamura $\mathrm{H}$, Terai $\mathrm{H}$, et al. Characteristic radiographic or magnetic resonance images of fresh osteoporotic vertebral fractures predicting potential risk for nonunion: a prospective multicenter study. Spine. 2011;36(15):1229-35.

4. Cooper C, Atkinson EJ, O'Fallon WM, et al. Incidence of clinically diagnosed vertebral fractures: a population-based study in Rochester, Minnesota, 1985-1989. J Bone Miner Res. 1992;7(2): 221-7.

5. Myers ER, Wilson SE. Biomechanics of osteoporosis and vertebral fracture. Spine. 1997;22(24):25S-31S.

6. Alexander EL, Malinow K, Lejewski JE, et al. Primary Sjögren's syndrome with central nervous system disease mimicking multiple sclerosis. Ann Int Med. 1986;104(3):323-30.

Spine Surgery and Related Research is an Open Access journal distributed under the Creative Commons Attribution-NonCommercial-NoDerivatives 4.0 International License. To view the details of this license, please visit (https://creativeco mmons.org/licenses/by-nc-nd/4.0/). 